\section{Magnitude estimation: Range of response and the exponent*}

\author{
ROBERT P. MARKLEYं \\ Fort Hays Kansas State College, Hays, Kans. 67601
}

Ss made magnitude-estimation judgments of the apparent distance of a space vehicle in a reduced cue setting. The effects of stimulus range on response range and the exponent of a Stevens-type power function were investigated. Limitations upon the generality of previous findings about the effects of this variable were discussed.

A fact clearly emerging from recent research in psychophysical scaling is that the exponent of a Stevens-type power function varies with changes in stimulus range. The numerical value of the exponent has been found to be sensitive to several second-order factors (Poulton, 1968). This variation limits the appropriateness of the power law as a model of sensory or perceptual processes. Also restricted is the usual interpretation that the exponent is a parameter indicative of the nature of the stimuli being judged. Poulton (1968), in a review of most of the available reports of range effects, estimates that about one-third of the variance of a set of published exponents can be accounted for by range alone. His first model suggests that Ss will increase their range of response with an increase in stimulus range, but that the increase is not of the same magnitude as the physical change.

Vincent. Brown, Markley, \& Arnoult (1968). while studying perceived distance, reported an additional range effect. These authors found. as had others before them. that the exponent decreased with an increase in stimulus range. But the response ranges used by Ss did not covary with the exponent or the stimulus range. There was an interaction between stimulus range and its location in the entire set of potential stimuli. The actual ranges of responses used were found to be more directly related to the number of discriminable stimuli within a physical range than to its absolute size.

The present study attempts to examine range effects further by independent manipulation of stimulus-range length and location. A within-Ss design examined the effects of these variables on the performance of the individual S. Distance judgments were obtained in the same

*This research and preparation of this paper was supported by Grant NGR 44009018 from the National Aeronautics and Space Administration to Texas Christian University, $M$. D. Arnoult. Principal Investigator. Additional support was provided by Grant PS6974 from the TCL Research Foundation.

-Address: Department of Psychology. Fort

Hay's Kansas State College. Kays. Kans. 67601. reduced cue setting simulating outer space as were the data of Vincent et al (1968). However, the target (object whose distance was to be judged) was different.

\section{SUBJECTS}

Twenty-four male Ss volunteered to serve in this study. All were paid and had no experience with psychophysical scaling research. Four Ss were familiar with the apparatus from a previous discrimination study. All Ss had 20/20 vision (or better), as determined by an examination conducted by an optometrist.

\section{APPARATUS}

Distance judgments were made in the NASA-TCU Space Vision Simulator (Arnoult, Vincent, Brown, Markley, \& Hensleigh. 1969). The simulator presents a high-fidelity three-dimensional representation of a space vehicle (in this study the Lunar Excursion Module, ascent phase) in a star-free setting otherwise simulating outer space. The appropriate object configuration, retinal image sizes, binocular cues, light-ray configurations, and relative brightness changes over a range of $45.7-6,096 \mathrm{~m}$ are presented by the simulator. Distance cues provided by terrain, context, texture gradients,
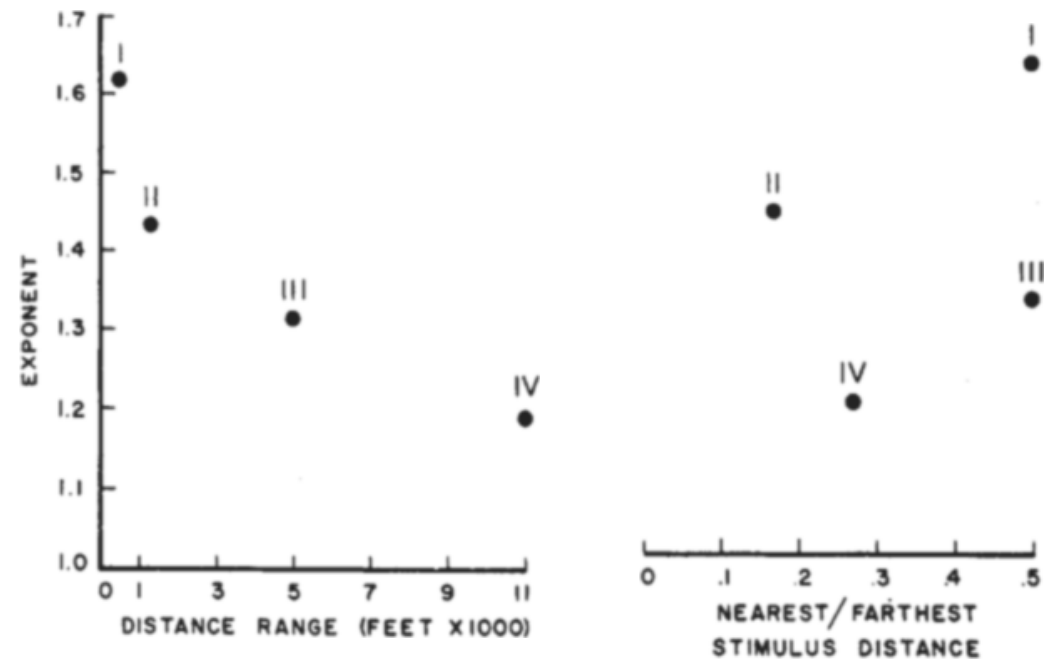

Fig. 1. Group exponents from psychophysical power functions as a function of two objective measures of range of stimulation. atmospheric haze, aerial perspective, and the like were absent. The ascent stage of the lunar module is a complex, irregularly shaped object, and at the time of the study was unfamiliar to the Ss. The module faced the $S$ and was oriented slightly to the left and away from the $S$. The simulated visible dimensions of the spacecraft were approximately $4.25 \mathrm{~m}$ wide at the base, $2.6 \mathrm{~m}$ wide at the top, and $2.7 \mathrm{~m}$ high.

STIMULI

Four sets of stimulus distances were used: 152.4, 170.7, 189, 213.4, 238, 274.3 , and $304.8 \mathrm{~m}$ for Range I; 76.2, 122 , $170.7,213.4,274.3,365.7$, and $457.2 \mathrm{~m}$ for Range II; 1524, 1707, 1890, 2134, 2380,2743 , and $3048 \mathrm{~m}$ for Range III; and $1220,1524,1829,2134,2743,3657$. and $4572 \mathrm{~m}$ for Range IV. The standard distance for Ranges I and II was $213.4 \mathrm{~m}$. The standard distance for Ranges III and IV was $2134 \mathrm{~m}$. Two independent variables, each with two levels, were manipulated in constructing the four stimulus ranges. The first variable was range location. Ranges I and II were near ranges, while Ranges III and IV were far ranges. The size of each range was the second independent variable. Ranges I and III were short ranges, while Ranges II and IV were long ranges.

The geometric lengths of Ranges II and IV were not set equal to each other. Range IV was shortened from $6: 1$ to $3.75: 1$. This was done to avoid using distances between 610 and $1220 \mathrm{~m}$. Previous identification trials had shown that Ss reported a phenomenological change in the character of the target between these distances. At distances shorter than $610 \mathrm{~m}$, Ss see a clearly identifiable spaceship. At distances beyond $1220 \mathrm{~m}$, most ubject characteristics are 


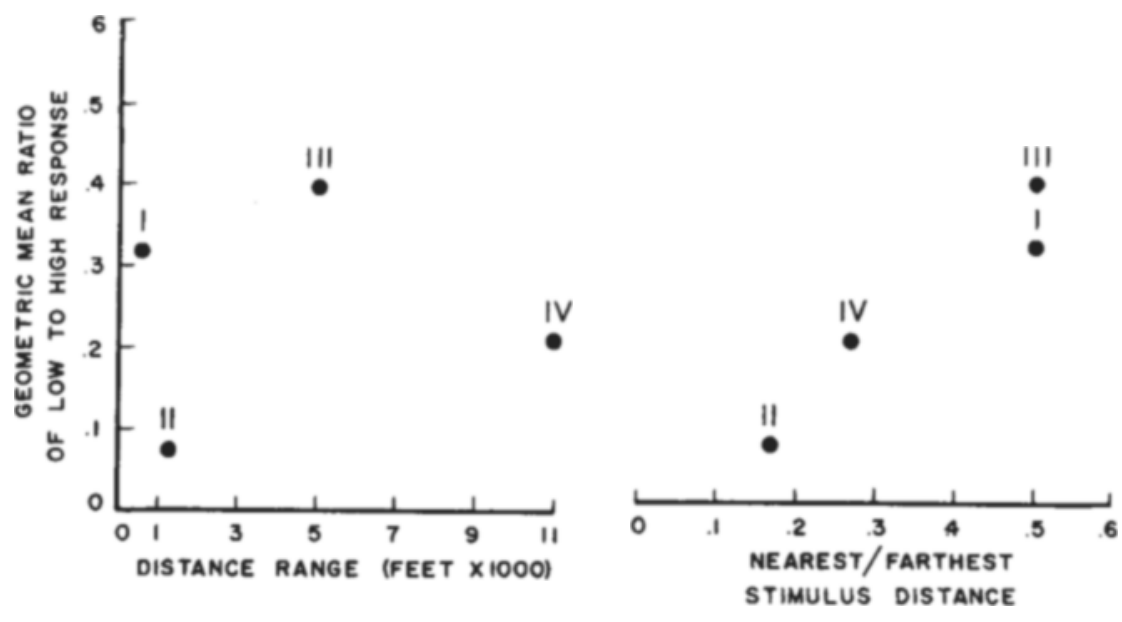

Fig. 2. Response range as a function of two measures of stimulus range.

lost, and Ss report seeing a pinpoint of light. Transition from one state to the other occurred in the range $610-1220 \mathrm{~m}$. The confounding of range length and location limits the use of statistical analyses of the results.

\section{PROCEDURE}

All Ss made magnitude estimation judgments of the distance of the module over the four ranges of distance. Each $S$ participated singly in two 40 -min sessions on consecutive days. Stimuli from two ranges were judged on each day. Each $\mathrm{S}$ judged the four ranges in a different order. Prior to the first day's distance judgments, $S$ made 20 magnitude estimations of the lightness (or darkness) of a set of Munsell neutral grays. This served to familiarize the $S$ with the task of making magnitude estimations. Ss were then brought into the observer's station of the simulator and informed about the nature and approximate size of the spacecraft. After a period of dark adaptation, Ss were allowed to view the module moving back and forth over a range of $76-4600 \mathrm{~m}$. Then the

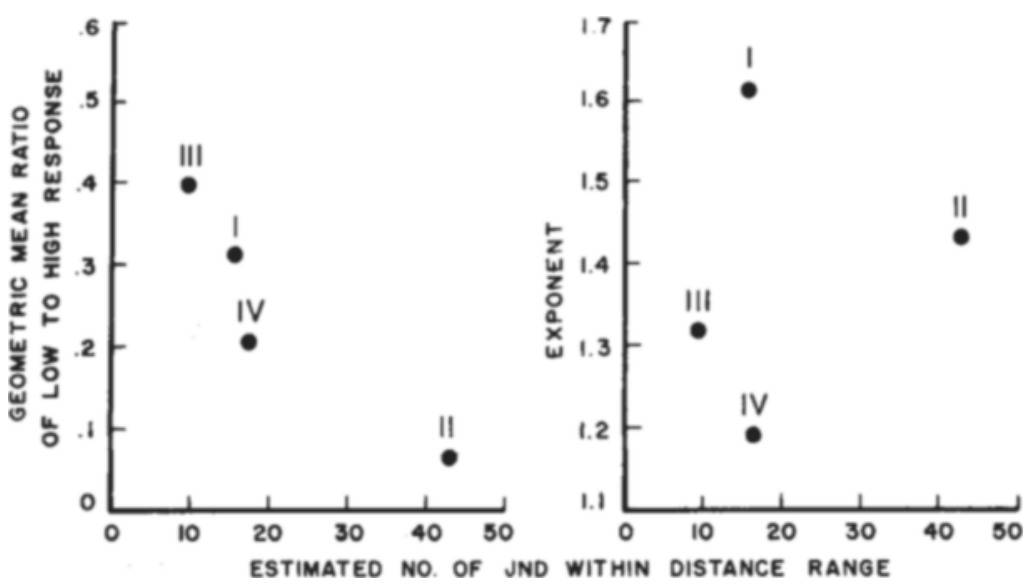

Fig. 3. Group response ratios and psychophysical exponents as a function of stimulus discriminability. for each $S$ and group. This was the ratio of each $S$ 's geometric mean response for the nearest stimulus in a range to his mean response for the farthest distance. The group geometric mean of the new statistic was then computed for each condition. Unlike the exponent, this ratio is a pure measure of range of response that is not biased by the physical measures used to describe the stimuli. A small ratio indicated a large range of responses.

The exponents, plotted as a function of range in Fig. 1, indicated, in accord with previous findings, that an increased range was associated with a lower exponent. The rank order of the exponents was the same as the rank order of the absolute physical ranges. It can also be seen that the far ranges had lower exponents than the near ranges. However, the exponents do not vary systematically with the dynamic ranges (ratio of smallest to largest distance) of the stimuli (Fig. 1b).

The values of these exponents were unusual. The group values are higher than previous group exponents obtained by Vincent et al (1968) from magnitude estimations of distance with the same apparatus and also higher than exponents of fractionation scales obtained by Markley, Brown. \& Arnoult (1968). This may be due to the unusual configuration of the target. There is some agreement with the results of Künnapas (1960), but no agreement with the exponents obtained by Künnapas (1968) in a reduced cue setting.

Furthermore, the interindividual variation in exponents is relatively large. Rule (1969) reported standard deviations of exponents from visual magnitude estimation tasks, ranging from .21 to .31 , whereas standard deviations for the present groups ranged from .49 to .69 .

Analyses of the response ratios are shown in Fig. 2. Stimulus range length can be seen to have affected range of response. A logarithmic transformation of the ratios as was used by Ekman et al (1968) did not alter the appearance of these results. Ss generally increased the range of numbers they used in responding as the length of the stimulus series relative to a particular constant standard increased. Shifting from a near to far set of distances appeared to decrease the range of numerical responses even though the absolute physical range increased.

The response ratios also varied directly with the approximate number of jnds contained in each stimulus set. The exponents did not vary with jnds (see Fig. 3). The number of jnds was obtained from the Weber function reported by Worley \& Markley (1969). It is interesting to note that, from the standpoint of discriminability, Ranges I and IV were 
nearly of equivalent length (Fig. 3). Yet, these two ranges produced the two extreme group exponents.

Stimulus range is a major contributor to group differences and to individual changes in performance on a psychophysical scaling task. The present data indicated that Ss adjust their response ranges with changes in stimulus range. However, the critical measure of stimulus range affecting response range appears to be the number of discriminable steps contained in the stimulus range rather than the absolute physical range. These results point to boundary conditions for Poulton's (1968) Model I. There are situations in which Ss will decrease their range of numerical responses in the face of a fivefold increase in physical range. Variation in subjective range may not show up as a variation in the power law's exponent when discriminability is variable over different portions of the plysical continuum.

REFERENCES

ARNOLLT, M. D.. VINCENT, R. J., BROWN, B.

R., MARKLEY, R. P.. \& HENSLEIGH, R. C.
A description of the NASA-TCL Space Vision Simulator. National Aeronautics and Space Administration CR $73305,1969$.

EKMAN. G.. HOSMAN, B., LINDMAN, R. LJLNGBERG, L.. \& ÁKESSON, C. A. Interindividual differences in scaling performance. Perceptual \& Motor Skills, 1968 , 26, 815-823.

KLNNAPAS, T. Scales for subjective distance. Scandinavian Journal of Psychology, 1960, 1 . 187-192.

KÜNNAPAS, T. Distance perception as a function of available visual cues. Journal of Experimental Psychology, 1968, 77, 523-529.

MARKLEY, R. P., BROWN, B. R., \& ARNOULT, M. D. Fractionation of distance in simulated space. National Aeronautics and Space Administration CR 73 306, 1969.

POLLTON, E. C. The new psychophysics: Six models for magnitude estimation. Psychological Bulletin, 1968, 69, 1-19.

RULE, S. J. Subject difference in exponents from circle size, numerousness, and line length. Psychonomic Science, 1969, 15, 284-285.

VINCENT, R. J., BROWN, B. R., MARKLEY, R. P., \& ARNOULT, M. D. Magnitude estimation of perceived distance over various distance ranges. Psychonomic Science, 1968, 13, 303-304.

WORLEY, J. K., \& MARKLEY, R. P. Distance discrimination in a reduced cue setting. Psychonomic Science, 1969, 17, 237-238.

\title{
Reaction time and short-term visual memory*
}

\author{
W. A. PHILLIPS and A. D. BADDELEY \\ Experimental Psychology Laboratory. University of Sussex \\ Brighton BN1 9QY, Sussex, England
}

Posner's method of using differences in RT for physical and name matches to estimate the time constant of visual STM is criticized as confounding the decay of the visual trace with the development of a name code. When this confounding is avoided by using stimuli that are hard to name (a 5 by 5 matrix of randomly filled squares), the time constant shown by both RT and errors is consistently longer than that reported by Posner.

Posner and his co-workers have recently devised an ingenious technique by which reaction-time (RT) measures may be used to study visual encoding, and from which they have drawn interesting conclusions about short-term visual memory. The technique involves presenting $\mathrm{S}$ with a letter, followed after a brief interval by a second letter which may be either the same or a different letter and may be either upper- or lowercase. When the second letter immediately follows the first. RT is

*The authors are grateful for financial support to the Medical Research Council. The computer facilities were made available through a grant from the Science Research Council.
(1969), and Dale \& Folarin (personal communication). Posner found little or no decay over a 20 -sec interval; Blick found decay continuing beyond $5 \mathrm{sec}$; and Dale and Folarin found decay continuing beyond $10 \mathrm{sec}$. Closer examination of the rationale behind the Posner result, however, suggests that it may be based upon a logical error. It assumes that the discrepancy between the RT to physically identical terms and terms with the same name but different case simply reflects the strength of the physical trace, with the two RTs becoming equal when the trace becomes indistinguishable from the visual background noise. It is clear from Posner's own experiments, however, that at the same time as the physical trace is fading, the item is being translated into a name code. The point at which the difference between the physical and name match RT disappears, therefore, represents the combined effect of a fading visual trace and a developing name code. Once the name code has developed to a point at which it allows faster RTs than the visual code, $\mathrm{S}$ will presumably use it in preference to the visual trace, even though visual trace continues to be available. Since $S$ need no longer use the visual trace, his RTs will no longer reflect its strength. In short, Posner's technique confounds the fading of the visual trace with the development of the name code, and as such cannot give a valid indication of the time course of visual STM.

This suggests that the method of Posner \& Keele (1967) may give an underestimate of the duration of visual STM, and that the longer times suggested by the other experiments are more accurate. However, the discrepancy may be due to differences in procedure. All three experiments suggesting longer times measured accuracy rather than reaction time, and studied memory for position or length rather than memory for form. Furthermore, Posner (1967) and Dale and Folarin used recall rather than recognition. The following experiment, therefore, studies visual STM using a technique analogous to that employed by Posner and Keele, but material which cannot easily be named in order to avoid the confounding of visual trace decay with name-code development. METHOD

Stimuli comprised a 5 by 5 matrix of squares in which each square had a 0.5 probability of being filled. A new pattern was used each trial. After a randomly selected delay of $0.3,1.0,3.0$. or $9.0 \mathrm{sec}$, a second matrix was presented. This was either identical to the first or differed by having one square more or one square less filled. Ss were instructed to decide "as quickly as possible without error." whether 